that obtained by the statistical method of Hauptman \& Karle (1953, p. 50). The connection between (17) and a Patterson-Harker section has already been pointed out by Vand \& Pepinsky (1953); it is mentioned here for the sake of completeness. Once again, the result will be of practical value only for simple crystal structures, or when the asymmetric unit includes some small number of approximately equal heavy atoms.

Similar results can be found for space groups which do not have a centre of symmetry, provided that they project to give a centrosymmetric plane group. For example, for space group $P 2$

$$
U(2 h, 0,2 l)=\overline{\left\{N U^{2}(h k l)-1\right\}},
$$

while for space group $P 2_{1}$

$$
U(2 h, 0,2 l)=N \overline{\left\{(-1)^{k} U^{2}(h k l)\right\}} .
$$

The same remarks apply to these two results as have been made about earlier ones.
It is interesting to note that for space groups $P 6_{1}$, $P 6_{1} 2$ and $R 3$ the magnitudes and signs of all structure factors in one zone can in principle be calculated from the general intensities. This is an immediate deduction from the work of Buerger (1946).

\section{References}

Buerger, M. J. (1946). J. Appl. Phys. 17, 579.

Cochran, W. (1953). Acta Cryst. 6, 810.

Cochran, W. \& Woolfson, M. M. (1954). Acta Cryst. 7, 450 .

HaUPTMAN, H. \& KaRLE, J. (1953). Solution of the Phase Problem. I. The Centrosymmetric Crystal. A. C. A. Monograph No. 3. Wilmington: The Letter Shop.

HUGHES, E. W. (1953). Acta Cryst. 6, 871.

SaYre, D. (1952). Acta Cryst. 5, 60.

VAND, V. \& PePinsky, R. (1953). The Statistical Approach to $X$-ray Structure Analysis. State College: The Pennsylvania State University.

VAND, V. \& Pepinsky, R. (1954). Acta Cryst. 7, 451.

Acta Cryst. (1954). 7, 583

\title{
A New Photographic Method for Studying the Texture of Large Single Crystals
}

\author{
By A. R. LANG* \\ Philips Laboratories, Inc., Irvington-on-Hudson, N.Y., U.S.A.
}

(Received 17 September 1953 and in revised form 19 April 1954)

\begin{abstract}
The orientation, in one plane, of macromosaic regions covering a crystal face several centimetres long may be determined from one photograph of the reflection pattern of characteristic radiation when the film-to-crystal distance is made large compared with that between crystal and X-ray source, and a grid placed in front of the film is rotated in synchronism with the crystal rotation. A line X-ray source parallel with the crystal rotation axis is used. The reflections are rapidly interpreted with the aid of a master pattern given by a good crystal which need not be of same material as the specimen.
\end{abstract}

\section{Introduction}

In order to assist the selection of suitable crystal specimens for use as monochromators it is desirable to develop methods for the X-ray examination of the reflecting planes which will give as much information as possible about the variation, over the surface of the plane, of the reflecting power, sharpness of reflection, and any large-scale misorientations that may be present. Good quality monochromators are required not only in diffraction work but also in the rapidly widening field of fluorescence analysis. Knowledge of the above properties also plays a part in studies of crystal growth and deformation.

A number of methods exist which will give some of the required data, though possibly only in qualitative

* Now at Division of Applied Science, Harvard University, Cambridge 38, Mass., U.S.A. form. The experimental arrangements of photographic methods using characteristic radiation differ with respect to the relative positions of $\mathrm{X}$-ray source, crystal, and film, and their relative motions, if any. They may be roughly classified according to whether the distance from source to crystal, $S$, is equal to, much less than, or much greater than $R$, the distance from crystal to film. When $S=R$ the Bragg focusing condition is obtained and the position at which the reflected ray strikes the film is, for moderate beam divergences, to a close approximation independent of the point on the crystal giving rise to the reflection; and though fluctuations in reflected intensity may be observed as the crystal is rotated through the beam, no information can be obtained about the distribution and orientation of the areas contributing reflections at any instant. When $S$ does not equal $R$ the position at which the reflected ray strikes the film is a function 
of both the location and orientation of the reflecting region. By making either $R \ll S$ or $R \gg S$ these factors may be separated. However, in the special case when the angular misorientations in the crystal are very small and information is required only about the variations in reflecting power, the ratio of these distances is not important; then, by placing a film parallel to the crystal face, and rotating it at the same rate as the crystal, an image of the face is obtained in which the intensity at any point corresponds to the reflecting power of the corresponding point on the crystal (Wooster \& Wooster, 1945).

When $R \ll S$ conditions correspond to those of the Berg (1931) technique, which has been successfully developed as a method for the microscopic study of crystal surfaces by Barrett (1945) and Honeycombe (1951). Provided that the film is placed sufficiently close to the crystal (this is not always possible if the crystal is large and the glancing angle high), the location of a diffracted ray on the film corresponds closely to the location on the crystal of the region giving rise to reflection, as long as the range of orientations is not so great that angular rotation of the diffracted beam causes a linear displacement on the film comparable with the distances along the crystal face which it is desired to resolve. When the angle subtended by the source at the crystal is kept low, not more than a few minutes of arc, by making the distance $S$ large, the relative orientations of different parts of the crystal plane can be determined by taking a number of 'stills' with the specimen rotated by a known amount between each exposure. The angular resolution of the method, for a given point on the crystal, depends upon the angle subtended by the source at the crystal. But if this angle is made quite small then some regions of the crystal may be missed unless an excessively large number of exposures be taken. This might occur with crystals of the sodiumchloride type in which individual regions have narrow rocking curves. For crystals in which the lattice may be subject to continuous curvature the method is more suitable. With a stationary crystal, the angle of incidence of the $X$-rays varies with position on the crystal surface, and hence allowance must be made for the linear separation, in the plane of incidence, of regions whose orientations are to be compared. This method has the advantage of giving a twodimensional picture, and when applied to metal single crystals shows clearly such features as slip bands, kink bands and polygon boundaries. When the angular range to be studied is quite small the resolution may be greatly enhanced by first reflecting the primary beam from a good quality single crystal (Bond \& Andrus, 1952).

As an alternative, the arrangement with $R \gg S$ may be adopted. With a stationary crystal, photographs may be taken which give information on the $\mathrm{X}$-ray emission-line shape and the degree of perfec- tion of the crystal (Valasek, 1930; Ramachandran, 1944).

For studying the whole face of the crystal a method has been developed (Lang, 1952) in which the crystal is rotated through an angular range sufficient to enable all regions in its surface to reflect the characteristic radiation, the reflected beams striking the photographic film in various positions as different regions pass through the reflecting angle. Provided that $R / S$ is not less than about 10, a quantitative interpretation of the pattern may be obtained quite simply. The crystal is placed as close to the $\mathrm{X}$-ray tube as possible, subject to the condition that the whole face be illuminated by the divergent beam, and a fine line $X$-ray focus is used parallel to the axis of rotation of the crystal. The position and instant at which a reflected ray strikes the film are a function of the tilt of the reflecting mosaic element and the inclination of the crystal to the primary beam, so if the relation between angular setting of the crystal and the time at which a reflection occurs is known, then both the position of the reflecting region and its inclination to a fixed direction (usually taken as that corresponding to the mean orientation of the crystal elements) may be calculated. For this purpose it is necessary to impress upon the photographic film a 'time-coordinate'. It must be done in such a way that the pattern of reflections is obscured as little as possible, and the coordinate must be made independent of position on the photographic film. One method of accomplishing this is to rotate a suitably designed grid of parallel opaque strips in front of a stationary film. From the slope of the shadows of the grid at any position on the film, the instant of reflection, and hence the angular setting of the crystal, may be found. Such use of a rotating grid was suggested by a technique devised by Orowan (1942). In practice a photograph can be interpreted by placing over it the pattern from a perfect, or nearly perfect, crystal. This master pattern is used to obtain rapidly the desired coordinates of mosaic element tilt and position. The chief limitation of this technique is the fact that mosaic tilts in only one plane can be measured, i.e. in the plane normal to the axis of crystal rotation, and that it is a one-dimensional picture of the crystal surface which is obtained. To meet these limitations it would be possible, first, to take a series of photographs with the crystal rotated by different amounts about the normal to the reflecting plane, and secondly, to build up from the patterns obtained from a series of narrow strips of the crystal surface a complete picture of the whole surface.

Recently a method has been developed by Schulz (1953) which gives an image of a crystal surface from which quantitative information on mosaic misorientations both parallel and perpendicular to the plane of incidence can be obtained. This employs white radiation and a micro-focus $\mathrm{X}$-ray tube, and is capable of high angular resolution. 


\section{Theory of the rotating-grid method}

The geometry of the method here described can be understood by reference to Fig. 1. The line focus is at $F$ and the crystal rotates about $C$ as axis. $F C D$ and $F A D^{\prime}$ are two rays reflected when the crystal face is in the settings $E C G$ and $A C B$ respectively, the crystal being rotated by an angle $\omega$ between these positions. Let the angular misorientation in the plane of the figure be $\psi$ at the point $A$, measured in the same sense as $\omega$. The orientation at $C$ is taken to define the direction with respect to which the angles $\psi$ are measured. The film $D D^{\prime}$ is perpendicular to the median ray $C D$ and distances, $x$, on the film are measured from $D$ in the direction of increasing diffraction angle. $F C=a$ and $C D=C F^{\prime}+F^{\prime} D=a+b$. From the figure it will be seen that the angle between the incident rays $F A$ and $F C$ is $\omega+\psi$; and, as the reflected rays cross approximately at the Bragg focus $F^{\prime \prime}, D D^{\prime}=$ $x \cong b(\omega+\psi)$. The closeness of this approximation may be now examined, remembering that $\omega$ is small and that $b \gg a$. It can be seen that $x=(a+b) \tan (\omega+\psi)$ less the projection parallel to $A D^{\prime}$ of $A C$ on $D D^{\prime}$. If the distance along the crystal of $A$ from $C$, measured from $C$, is $p$, then

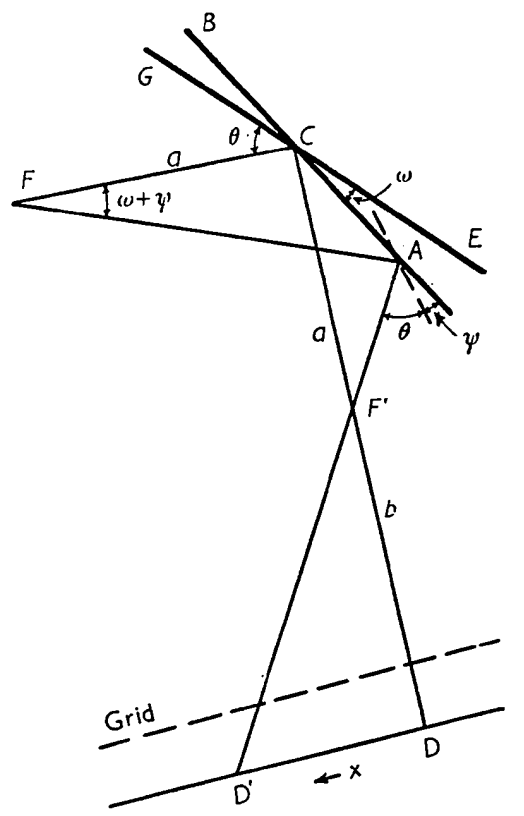

Fig. 1. Geometry of rotating-grid technique.

$$
x=(a+b) \tan (\omega+\psi)-p \sin (\theta+\psi) \sec (\omega+\psi) .
$$

In the triangle $A F C$,

$$
p=a \sin (\omega+\psi) / \sin (\theta-\psi) .
$$

Substituting for $p$ in equation (1), neglecting products of small quantities, and putting $\sin \omega=\omega, \sin \psi=\psi$, $\cos \omega=\cos \psi=1$, there is obtained

$$
\begin{gathered}
x=b(\omega+\psi)\{1-2 \psi(a / b) \cot \theta\} \\
\simeq(\omega+\psi) b .
\end{gathered}
$$

In the special case when $\psi=0$, it follows that

$$
x=\omega b \text {. }
$$

To find the relation between $x$ and $p$, the value of $(\omega+\psi)$ given by $(3)$ is substituted in (2). There is then obtained, after expanding $\sin (\theta-\psi)$,

$$
\begin{aligned}
p & =\frac{a x}{b \sin \theta}\{1+\psi \cot \theta(1+2 a / b)\} \\
& \cong a x / b \sin \theta .
\end{aligned}
$$

It will be noted that this relation 2 independent of $\psi$. The crystal faces studied have been about $3 \mathrm{~cm}$. long, so the limits of $p$ are $\pm 1.5 \mathrm{~cm}$. The value of $\psi$ does not generally exceed the limit. $\pm 1^{\circ}$. With commonly used reflecting planes, crystal dimensions, and crystal and film positions, approximate equation (6) gives values of $p$ accurate to about $5 \%$, and (4) is correct within $1-2 \%$.

Now it is required to find the value of $\psi$ for every value of $p$ on the crystal face, the measured quantity being the slope of the shadow, $\eta$, at a point on the film distant $x$ from $D$. From (6) $p$ is found directly from $x$, and $\psi$ can be found from $\eta$ if the relation is known between angular settings of the grid and of the crystal. The procedure is much simplified if a pattern given by a good quality crystal is available. Suppose the slope $\eta$ is the function of $\omega$ denoted by $\eta=K(\omega)$. For the good crystal pattern, at the point $x^{\prime}, K(\omega)=$ $K\left(x^{\prime} \mid b\right)$, from (5). The value of $\omega$ at the point $x$ on the specimen pattern is that for which $\eta$ is the same on the good crystal pattern, the 'master' pattern, i.e. $\omega=x^{\prime} \mid b$. This may be substituted in the expression for $\psi$ obtained from (4),

giving

$$
\psi=\frac{1}{b}(x-b \omega),
$$

$$
\psi=\frac{1}{b}\left(x-x^{\prime}\right) .
$$

The method may be illustrated by an application to the study of the cleavage face of a lithium fluoride plate. The master pattern (Fig. 2(a)), given by a crystal consisting of a single reflecting region, shows a regular cycloidal shadow pattern. This may be compared with the discontinuous pattern (Fig. 2(b)) produced by the specimen crystal which contained a number of reflecting regions, tilted at various angles to each other. The face of the specimen crystal was $2.1 \mathrm{~cm}$. in length, so, with $a=18 \mathrm{~cm}$. and $b=180$ $\mathrm{cm}$., the pattern produced by the 200 reflection of $\mathrm{Cu} K \alpha$ radiation from this face covered a length of $7.5 \mathrm{~cm}$. on the film, in accordance with $(6)$.

From the geometrical relations given above it is easy to calculate suitable distances $a$ and $b$ for the study of any particular crystal face. The source-tocrystal distance $a$ should be sufficient to ensure that the whole length of crystal surface under examination 
is illuminated. With standard X-ray tubes, allowing beam divergences of $6^{\circ}$ or more, this condition is usually satisfied with values of $a$ less than $20 \mathrm{~cm}$. The limit to the length of crystal face that can be

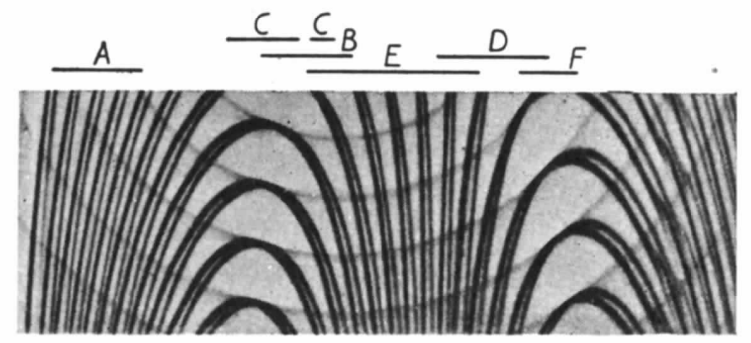

(a)

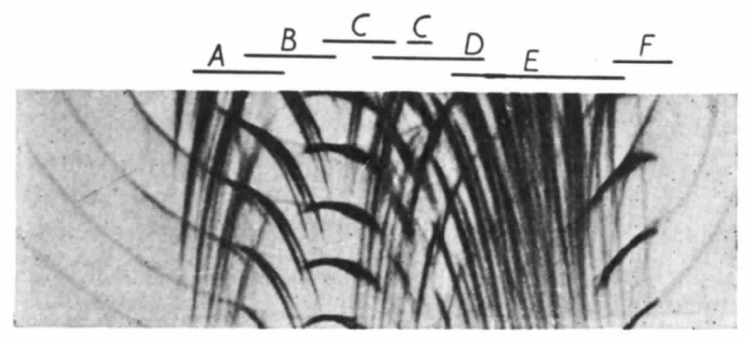

(b)

Fig. 2. (a) Master crystal pattern, (b) specimen crystal pattern. $\mathrm{LiF}$ cleavage face, 200 reflection, $\mathrm{Cu} K \alpha$ radiation.

studied in one exposure is fixed, as long as the condition $b / a \leqslant 10$ is maintained, by the size of film (and grid) and by the Bragg angle, as can be seen from (6). The length $a$ will also determine the range of $\omega$ needed to bring all crystal regions into the reflecting position. It is desirable to choose $a$ and $b$ so that the $K \beta$ reflection pattern does not overlap that due to $K \alpha$. It is then not necessary to use filtered radiation.

The technique for interpretation of the specimen pattern will be described below.

\section{Design of rotating grid}

Two rotating grids have been used. The first was very simply constructed from four $\frac{1}{2}$ in. $\times \frac{1}{2}$ in. angle members bent from 20 s.w.g. tin plate and spot-welded together. A bisecting member, also of $\frac{1}{2}$ in. $\times \frac{1}{2}$ in. angle, carried a central bushing which fitted on the shaft of a synchronous clock motor. The area of the square, 12 in. $\times 12$ in., was filled with a number of 24 s.w.g. tin-plate strips spot-welded in position. The second grid was in the form of a brass disc in which a number of parallel slots were cut. The disc was mounted in a light-alloy ring. Three small wheels supported the ring by engaging a groove in its periphery. Two of the wheels, mounted on ball bearings, idled, while the third wheel, fitted with a hard rubber tire, was positively driven through a variable gear train by a synchronous motor. The effectively used diameter of this grid was about 6 in.

Two points need to be observed in the design of grids. The ratio of slot width to opaque strip width, and the spacing between slots, should be chosen, without making this interval too large, to give a clear record avoiding confusion due to the $K \alpha$ doublet. This condition was reasonably satisfied with the second grid, with which the patterns of Fig. 2 were obtained.

The diameter of the grid should be made sufficiently large so that the influence of the grid center in modifying the form of the cycloidal pattern is as small as possible. The second, smaller, grid was inferior to the larger grid in this respect.

A grid rotation speed of $0.5-1$ revolution per hour was usually employed. It should be remembered that the cycloidal pattern repeats after half a grid revolution. The usual crystal rotation speeds were $\frac{1}{8}$ or $\frac{1}{1 \frac{1}{6}}$ of a degree $\omega$ per minute.

\section{Method of interpretation of patterns}

The following technique for interpretation was adopted. The specimen pattern Fig. 2(b) was fixed to a viewing screen and the master pattern Fig. 2(a) was placed over it. From the extent of individual lengths along the specimen film over which coincidence with the master pattern could be obtained, the limits of the various reflecting regions, $A, B, C$, etc. on the crystal could be determined, using the relation between $x$ and $p$. A scale was attached to the master film and the zero value of $\psi$ was taken when the two patterns were in coincidence over a certain region in the centre of the crystal, say region $C$. In this position a marker was fixed on the viewing screen opposite the zero of the scale on the master pattern. Then the master pattern was shifted until coincidences were found for the other regions of the crystal, the values of $x-x^{\prime}$ being given directly by the relative displacement of specimen and master films, as read from the scale on the latter. The sections of the master pattern with which sections of the specimen pattern were matched are lettered correspondingly. The resulting values of $\psi$, derived from equation (7), can be plotted against $x$ (or $p$ ) to give a clinometric traverse, or clinogram, of the crystal face, that obtained from Fig. 2 being shown in Fig. 3. When more than one region is included in the height of crystal face studied at a given value of $p$,

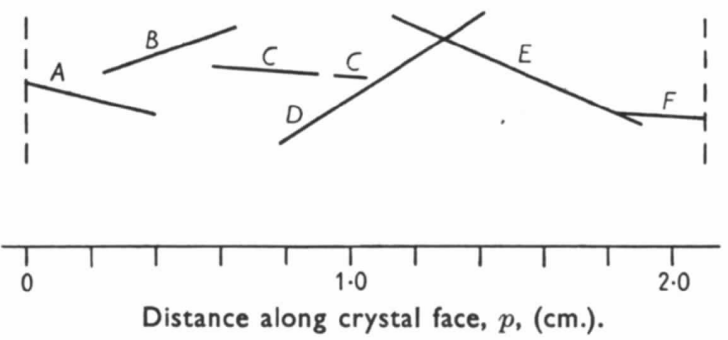

Fig. 3. Clinogram of lithium fluoride cleavage face. 
slopes for these are plotted separately. The angular scale is exaggerated fiftyfold in the clinogram; thus a slope of $1^{\circ}$ in the clinogram represents $0.02^{\circ}$ in the crystal face.

Relative orientations only were found in this experiment and the results were plotted in such a way as to show the average slope to be approximately zero. Absolute measurements of $\psi$ could of course be made if for both records $\eta$ was the same for a given value of $\omega$. It should be noted that when solely relative measurements are required the only data needed, apart from the fixed dimensions $a$ and $b$, are the values of $x-x^{\prime}$. It is not even necessary for rotation of the crystal or grid to be at a uniform rate; all that is required is that movements should be the same during the recording of both master and specimen patterns. One master pattern will serve for all reflections. This follows since equation (7) does not involve $\theta$ and $p$, and it can be seen from the consideration that the form of the relationship $\eta=K(\omega)$ depends only upon the relative rotation speeds of grid and crystal. It is necessary to ensure that the angular equivalent of the repeat distance along the film of the master pattern exceeds twice the range of angles $\psi$ : if this condition is not fulfilled, the possibility of ambiguity in interpretation of the specimen pattern arises. For best results with the method, the ratio of rotation speeds should be chosen to make this angular equivalent as small as possible, compatible with the above-mentioned condition, in order to gain the highest sensitivity in the measurement of $\psi$. A particularly bad crystal has been chosen to illustrate the method in Figs. 2 and 3, but it will be observed that though the total range of $\psi$ is about $1^{\circ}$, individual regions give very sharp reflections. It is for crystals of this type that the method is especially suitable.

The good resolution of the $K \alpha$ doublet shows that the individual regions have rocking curves less than $30^{\prime \prime}$ of arc in width. In the complicated pattern of Fig. $2(b)$ the accuracy of measurement of $\psi$ depends upon how closely the translation $x-x^{\prime}$ can be estimated, and in this particular case is probably not better than $6^{\prime}$ of arc. However, in a better crystal, breaks in individual arcs can be observed corresponding to small misorientations. An example of this can be seen on the right of Fig. 2(a); the displacement corresponds to a relative misorientation of about $2^{\prime}$ of arc.

Absolute angular rotation speeds are chosen to give an adequately dense exposure during one sweep of the crystal through the reflecting range. The time taken for this was about $1 \mathrm{hr}$. in the case of Fig. $2(a)$ and about $40 \mathrm{~min}$. in the case of Fig. $2(b)$.

For the study by transmission of the sharpness of reflection and of the orientation range of reflecting regions there can be employed the method of focusing
Laue spots (Guinier \& Tennevin, 1949). The method here described may also be used for transmission crystals, with small modification to the formulae. It allows, moreover, the position and orientation of each reflecting region to be found.

In the case of symmetrical transmission, equations (6) and (7) become,

and

$$
p=\frac{x a}{(2 a+b) \cos \theta}
$$

$$
\psi=\frac{1}{(2 a+b)}\left(x-x^{\prime}\right),
$$

the distances from source to crystal and crystal to film remaining $a$ and $a+b$ respectively.

\section{Conclusion}

The chief features of the method may be summarized as follows:

\section{Advantages}

1. Complete information is obtained concerning the position and orientation of crystal regions, in one plane and along one direction on the crystal surface.

2. High angular resolution of diffracted rays is achieved, hence full information is given on the crystal type, i.e. amount of lattice curvature, sharpness of rocking-curve peaks.

3. The method is quantitative yet the interpretation is quite simple.

4. The method is automatic and one film only is required to obtain the above-mentioned information.

\section{Disadvantage}

Each experiment gives a picture of the texture variation in one dimension only. However, this limitation is not serious in the study and selection of monochromator crystals.

\section{References}

Barretr, C. S. (1945). Trans. Amer. Inst. Min. (Metall.) Engrs. 161, 15.

Berg, W. F. (1931). Naturwissenschaften, 19, 391.

Bond, W. L. \& Andrus, J. (1952). Amer. Min. 37, 622. Guinier, A. \& Tennevin, J. (1949). Acta Cryst. 2, 133. HoNeYCOMBe, R. W. K. (1951). J. Inst. Metals, 80, 39. LaNG, A. R. (1952). Ph.D. Dissertation, Cambridge. Orowan, E. (1942). Nature, Lond. 149, 355.

Ramachandran, G. N. (1944). Proc. Indian Acad. Sci. $A, 20,245$.

Schulz, L. G. (1953). Paper No. 73, A. C. A. Summer Meeting.

VAlASEK, J. (1930). Phys. Rev. 36, 1523.

Wooster, N. \& Wooster, W. A. (1945). Nature, Lond. 155, 786 . 